\title{
Measuring Intraocular Pressure: How Important is the Central Corneal Thickness?
}

\author{
Sushmita Kaushik, Surinder Singh Pandav \\ Advanced Eye Centre, Postgraduate Institute of Medical Education and Research, Chandigarh, India \\ e-mail: sushmita_kaushik@yahoo.com
}

\section{INTRODUCTION}

Measurement of intraocular pressure (IOP) is fundamental to the management of glaucoma since it remains the only modifiable risk factor. Recent studies, especially on ocular hypertensives $(\mathrm{OHT}),{ }^{1-3}$ have stressed upon the influence of central corneal thickness (CCT) on IOP measurement. It is now quite well recognized that abnormally thick or thin corneas give rise to fallacious IOP measurement. Recent evidence suggests that it is not the central corneal thickness alone, but that other factors of corneal biomechanics also matter in influencing the IOP measurement.

This review will look at the importance of central corneal thickness in the management of glaucoma and glaucoma suspects. We will also discuss other corneal factors such as elasticity and corneal hysteresis, and how new technology promises to measure IOP taking these factors into consideration.

\section{WHY IS IOP MEASUREMENT BY GAT NOT PERFECT?}

The CCT affects the accuracy of IOP measurement by applanation tonometry, because the assumptions of the basic technique of tonometry are not met. Briefly, Goldmann applanation tonometry (GAT), which is the current gold standard for measuring IOP, is based on the Imbert-Fick's law. This states that the pressure within an infinitely thin, dry, smooth-walled, flexible sphere is equal to the external force required to flatten the surface of the sphere divided by the area flattened. However, these assumptions are made to the cornea, which is actually neither infinitely thin, nor its surface dry. In
1957, Goldmann and Schmidt ${ }^{4}$ deduced that for an average corneal thickness of 520 microns, the force of surface tension caused by the tear film was counteracted by the opposite force of corneal elasticity when the flattened area was $3.06 \mathrm{~mm}$.

\section{WHAT IS THE "NORMAL” CCT?}

Professor Goldmann, at the time of designing the GAT assumed that in non-diseased corneas, the thickness would not vary much from 520 microns. However, it has since been shown that "normal CCT" varies between ethnic groups ${ }^{5-9}$ and, indeed varies considerably within any normal population. In a metaanalysis of CCT in eyes designated normal, ${ }^{10}$ the mean CCT was $536 \pm 31$ microns. Slit-lamp based pachymetry values were slightly less than ultrasonic measurements ( $530 \pm 29$ microns vs $544 \pm 34$ microns respectively).

\section{CCT DISTRIBUTION IN OCULAR HYPERTENSIVES AND NORMALS}

In a study in Indian eyes, ${ }^{11}$ the authors found that the normal curve of CCT for normal patients was between 500 and 550 microns, while the normal distribution in ocular hypertensives was markedly skewed to the right (Table 1, Fig. 1). This also indicates the fallacious labeling of ocular hypertensives in individuals who are normal but for their uncorrected IOP measurement.

\section{RELEVANCE OF CCT IN IOP MEASUREMENT}

IOP is overestimated in thicker corneas and underestimated in thinner ones. However, the relationship has not been precisely

Table 1: Mean CCT values in normal and ocular hypertensive individuals

\begin{tabular}{llllll}
\hline Diagnosis & Mean \pm SD & $\begin{array}{l}\text { CCT }(\mu) \\
\text { Median }\end{array}$ & Min & Max \\
\hline Normal & $542.3 \pm 21.5 \#$ & 548.0 & 534.9 and 549.7 & 475 & 575 \\
OHT & $565.2 \pm 41.4 \#$ & 563.0 & 553.5 and 576.8 & 484 & 690 \\
\hline
\end{tabular}

Courtesy: Kaushik et al. Am J Ophthalmol 2006 

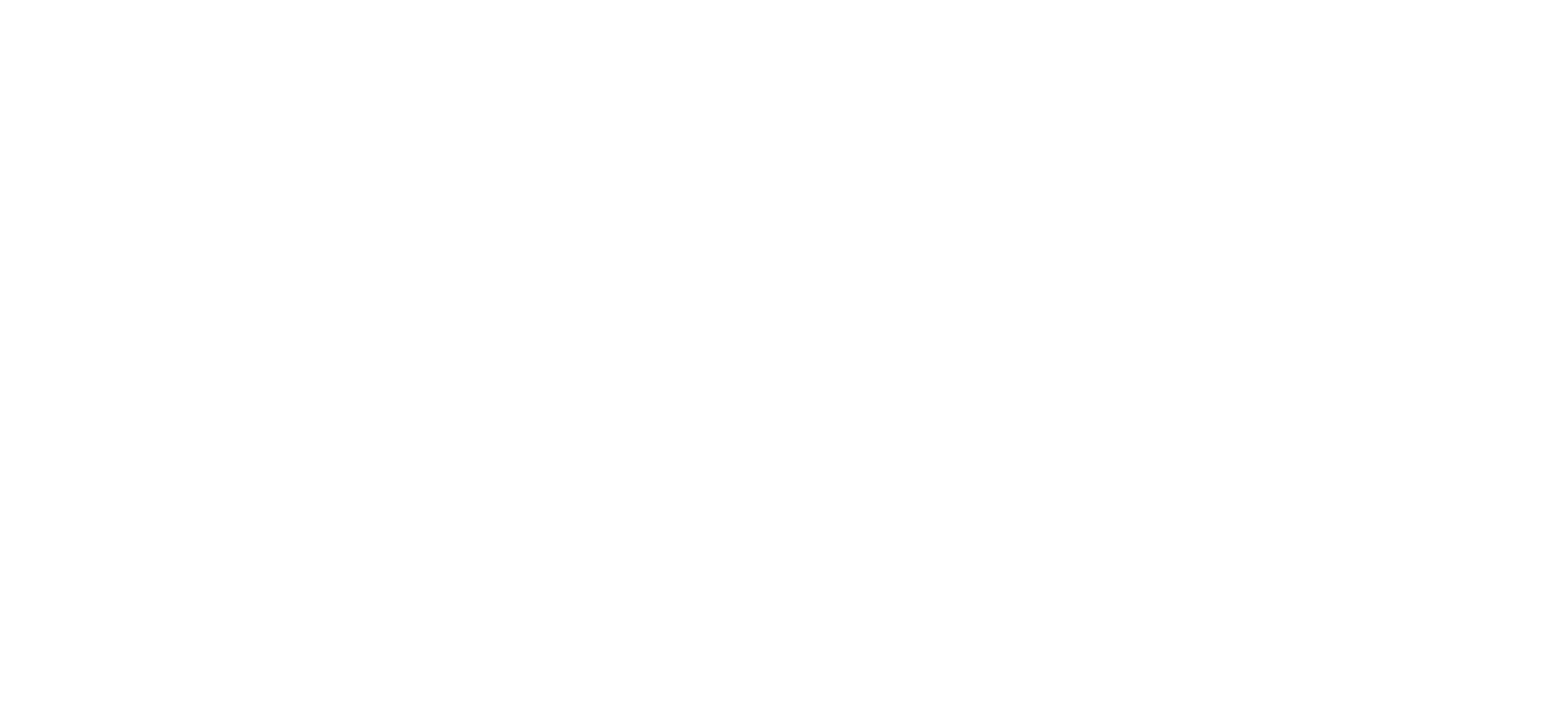

Fig. 1: Distribution of central corneal thickness in normal and ocular hypertensive individuals (Kaushik et al. Am J Ophthalmol 2006)

specified, and may or may not be linear. In 1975, Ehlers ${ }^{12}$ reported a manometric study on 29 eyes compared to Perkin's tonometry. Applanation tonometry agreed with manometric readings only at a CCT of 520 microns. He noted the relation of central corneal thickness to IOP in glaucomatous eyes to be as much as $7 \mathrm{~mm}$ Hg per 100 microns. A more recent meta-analysis ${ }^{10}$ of 133 data sets estimated the mean relationship to be $3.4 \pm 0.9$ $\mathrm{mm} \mathrm{Hg}$ for every 10 percent change in CCT.

However, it is not the CCT alone which would affect the IOP measurement. Corneal rigidity plays an important role. In clinical situations, corneal edema, with excessive hydration of the stroma, would give rise to falsely low IOP reading, even though the central corneal thickness would be increased. This is simply because it would be easier to applanate the softer edematous cornea, and consequently the end point would be reached earlier than usual. Similarly, a central corneal scar would result in a thinner cornea in the region of the scar tissue, but the greater tissue rigidity would give rise to falsely high IOP measurement.

\section{Physiological Variability of the CCT}

The CCT is a relatively stable measurement, but there are certain conditions which give rise to its variability, which must be kept in mind. Physiologically, the CCT may be increased immediately after waking up due to overnight corneal hydration. Peak circadian CCT values have been reported at $4 \mathrm{am} .{ }^{13}$ It is therefore advisable to measure the CCT at least 2 hours after waking. CCT may also be increased with chronic contact lens wear and dry eye.

\section{Racial Variation}

Several studies ${ }^{5-7}$ have found that African-Americans have thinner corneas on average than Caucasians, Hispanics and Asians. Though POAG is reported to be four times more prevalent among African-Americans than among Caucasians, ${ }^{8,9}$ it is unclear whether the thinner CCT is responsible for this increased risk of POAG.

\section{Effect of Refractive Surgery}

With the increasing incidence of refractive surgery procedures, it is important to be aware of pre-procedure corneal thickness measurements. It is inadvisable to use only the CCT to correct for IOP since the corneal biomechanics also changes after these procedures. The structural change is unpredictable, so there is no linear relationship of the post-procedure CCT to the IOP. After myopic LASIK or photorefractive keratectomy (PRK), the CCT decreases, and the measured IOP decreases by 1 to $2 \mathrm{~mm}$ Hg. After hyperopic LASIK or PRK, the measured IOP also drops by 1 to $2 \mathrm{~mm} \mathrm{Hg}$ with negligible change in CCT. Therefore, it is more useful to carry a card with key pre-LASIK data including IOP and CCT. Since the IOP recording may be inaccurate in the setting of refractive surgery, it is very important to ensure careful disk examination in these patients, especially those with borderline IOP measurements.

\section{PROGNOSTIC VALUE OF THE CCT}

Both the Ocular Hypertensive Treatment Study and the European Glaucoma Prevention Study showed that thinner 
corneas had an independent risk factor in predicting the progression of ocular hypertensives to primary open-angle glaucoma.

Recent studies also demonstrated that ocular hypertensives with abnormal Frequency Doubling Perimetry (FDP) ${ }^{14}$ or Short Wave Automated Perimetry (SWAP) ${ }^{15}$ results had thinner corneas, indicating that they may actually be individuals with "pre-perimetric" glaucoma which had not been detected by white-on-white standard automated perimetry.

\section{CCT AS STRUCTURAL CORRELATE FOR GREATER SUSCEPTIBILITY TO GLAUCOMA}

The CCT has also been thought to be a marker of the structural properties of the eye as a whole, including the retinal nerve fiber layer and lamina cribrosa. In a study ${ }^{11}$ of RNFL thickness measurements by optical coherence tomography (OCT) in ocular hypertensives, the authors found that ocular hypertensives with thin corneas had thinner RNFL measurements compared to OHTs with thick corneas and normal subjects. Similar findings were reported by Henderson et al ${ }^{16}$ using the GDx for RNFL measurements.

In a study on patients with primary open-angle glaucoma (POAG) with 35 percent reduction in IOP, Lesk et al ${ }^{17}$ demonstrated that patients with thinner corneas showed significantly greater lamina cribrosa displacement on the HRT. Whether this indicates a more mobile lamina cribrosa in patients with thinner corneas, and what implications it has for glaucoma, remains unclear as yet.

\section{BEYOND THE CCT: HYSTERESIS AND ELASTICITY}

Other factors of corneal biomechanics apart from the CCT are now thought to affect IOP measurements. Whereas CCT has been widely studied, it is likely that other factors, including corneal hydration, ${ }^{18}$ and bioelasticity, all determine to some extent the response of the corneoscleral shell to the force applied during the measurement of IOP. When stress is applied and removed, elastic materials follow the same path during deformation and relaxation, ultimately recovering their original shape. Viscoelastic materials are also able to recover their original shape after stress is removed, but the relaxation path is different from the deformation path. This behavior is referred to as hysteresis and is due to the dissipation of energy as heat within the material.

\section{Measurement of Hysteresis}

The Reichert Ocular Response Analyzer (ORA; Reicher Ophthalmic Instruments, Depew, New York) is a relatively new NCT device that measures IOP using a bidirectional applanation method. It determines IOP and corneal hysteresis during rapid motion of the cornea in response to a short duration (20 ms) air impulse. The air impulse causes the cornea to move inward, through applanation, and into slight concavity. Milliseconds after applanation, the air pump shuts off and the cornea moves through a second applanation while returning from concavity to its normal convex curvature. The difference of the two applanation event pressures is determined by viscoelastic properties of the corneoscleral shell. ${ }^{19}$ The rapid motion of the cornea during deformation creates velocity (rate)-dependent forces that oppose the forces (pressure) created by the air impulse. These opposition forces absorb energy from the air impulse, causing time delays (hence the term "hysteresis") in the occurrence of the applanation events. These time delays cause the inward and outward applanation event pressures to increase and decrease, respectively. Thus the difference in the pressures reflects a viscoelastic biomechanical property of the cornea, which is termed hysteresis.

Recently, there has been interest in the possible association between corneal hysteresis and glaucoma risk. Luce and Taylor ${ }^{20}$ found that patients with normal tension glaucoma and primary open angle glaucoma had lower corneal hysteresis. They have hypothesized that this may be related to corneal remodeling. In another study, Congdon et $a l^{21}$ found that low corneal hysteresis was predictive of visual field progression in patients with glaucoma.

\section{THE CLINICAL UTILITY OF KNOWING THE CENTRAL CORNEAL THICKNESS}

The CCT is undoubtedly of value in management of both glaucoma and glaucoma suspects, especially occur hypertension. However, in the absence of a well-established nomogram to "convert" IOP readings according to the CCT, it may be prudent to be able to classify the cornea into thin, average or thick category, and base management decisions accordingly. Some practical questions about the CCT which arise during clinical practice are summarized in Box 1 below.

\section{Box 1: Practical questions about CCT}

1. For which patient is pachymetry appropriate?

- Preferably all patients presenting to a glaucoma clinic

- ALL glaucoma suspects.

2. Why?

- Significant deviation from population mean may change management strategy

- OHT with thick corneas

- $\quad$ Thin corneas with borderline IOP in disk or field suspects.

3. What is the optimal way to measure CCT?

- Ultrasonic pachymetry

- Should be done early in examination

- The probe should be centered on cornea 
Contd...

- Take care to measure at least 2 hours after awakening

- Consider repeating at least once on different visit

- Look out for confounders-contact lens wear, corneal edema, dry eye.

\section{What correction factor should be applied?}

- Though many different algorithms have been published, there is no uniformly accepted correction factor for IOP according to the CCT

- A meta-analysis of many different populations deduced $2.5 \mathrm{~mm} \mathrm{Hg}$ for every $50 \mu$ increase in CCT

- It may be therefore useful to just document the (uncorrected) IOP reading and note the CCT in brackets adjacent to it-IOP mm $\mathrm{Hg}(\mathrm{CCT} \mu)$

- It is better not to record "corrected” IOP readings.

\section{SUMMARY}

Central corneal thickness is important to complete the overall picture of the individual patient. It helps tailor management decisions especially in borderline suspects and ocular hypertensives. Actual IOP correction appears unnecessary in each patient, since we would only be attempting to correct for an already imprecise measurement, given the many assumptions made about the cornea while measuring IOP by GAT. It may be clinically more useful to characterize cornea as "thin" "average" or "thick." However, with the recognition of corneal biomechanical properties other than the structural corneal thickness alone, newer measurement tools such as the Ocular Response Analyzer, which measures corneal hysteresis, may provide answers hitherto missed by applanation tonometry alone.

\section{REFERENCES}

1. Gordon MO, Beiser JA, Brandt JD, et al. The ocular hypertension treatment study: baseline factors that predict the onset of primary open-angle glaucoma. Arch Ophthalmol 2002;120:71420; discussion 829-30.

2. Brandt JD, Beiser JA, Kass MA, Gordon MO. Central corneal thickness in the ocular hypertension treatment study (OHTS). Ophthalmology 2001;108:1779-88.

3. European Glaucoma Prevention Study (EGPS) Group. Predictive factors for open-angle glaucoma among patients with ocular hypertension in the European glaucoma prevention study. Ophthalmology 2007;114:3-9.

4. Goldmann H, Schmidt T. Applanation tonometry. Ophthalmologica 1957;134:221-42.

5. Nemesure B, Wu SY, Hennis A, Leske MC for the Barbados Eye Study G. Corneal thickness and intraocular pressure in the Barbados Eye Studies. Arch Ophthalmol 2003;121:240-4.

6. La Rosa FA, Gross RL, Orengo-Nania S. Central corneal thickness of Caucasians and African-Americans in glaucomatous and nonglaucomatous populations. Arch Ophthalmol 2001;119:2327.

7. Shimmyo M, Ross AJ, Moy A, Mostafavi R. Intraocular pressure, Goldmann applanation tension, corneal thickness, and corneal curvature in Caucasians, Asians, Hispanics, and AfricanAmericans. Am J Ophthalmol 2003;136:603-13.

8. Tielsch JM, Sommer A, Katz J, et al. Racial variations in the prevalence of primary open-angle glaucoma: the Baltimore Eye Survey. JAMA 1991;266:369-74.

9. Sommer A, Tielsch JM, Katz J, et al. Racial differences in the cause-specific prevalence of blindness in east Baltimore. N Engl J Med 1991;325:1412-7.

10. Doughty MJ, Zaman ML. Human corneal thickness and its impact on intraocular pressure measures: a review and metaanalysis approach. Surv Ophthalmol 2000;44:367-408.

11. Kaushik S, Gyatsho J, Jain R, Pandav SS, Gupta A. Correlation between retinal nerve fiber layer thickness and central corneal thickness in patients with ocular hypertension: an optical coherence tomography study. Am J Ophthalmol 2006;141:88490.

12. Ehlers N, Bramsen T, Sperling S. Applanation tonometry and central corneal thickness. Acta Ophthalmol (Copenh) 1975;53:34-43.

13. Fogagnolo P, Rossetti L, Mazzolani F, Orzalesi N. Circadian variations in central corneal thickness and intraocular pressure in patients with glaucoma. Br J Ophthalmol 2006;90(1):24-8.

14. Medeiros FA, Sample PA,Weinreb RN. Corneal thickness measurements and frequency doubling perimetry abnormalities in Ocular Hypertensive eyes. Ophthalmology 2003;110:19038.

15. Medeiros FA, Sample PA,Weinreb RN. Corneal thickness measurements and visual function abnormalities in ocular hypertensive eyes. Am J Ophthalmol 2003;135:131-7.

16. Henderson PA, Medeiros FA, Zangwill LM, Weinreb RN. Relationshiop between central corneal thickness and retinal nerve fiber layer thickness in ocular hypertensive patients. Ophthalmology 2005;112:251-6.

17. Lesk MR, Hafez AS, Descovich D. Relationship between central corneal thickness and changes of optic nerve head topography and blood flow after intraocular pressure reduction in openangle glaucoma and ocular hypertension. Arch Ophthalmol 2006;11:1568-72.

18. Dayanir V, Sakarya R, Ozcura F, et al. Effect of corneal drying on central corneal thickness. J Glaucoma 2004;13:6-8.

19. Soergel F, Jean B, Seiler T, et al. Dynamic mechanical spectroscopy of the cornea for measurement of its viscoelastic properties in vitro. Ger J Ophthalmol 1995;151-6.

20. Luce D, Taylor D. Reichert ocular response analyzer measures corneal biomechanical properties and IOP provides new indicators for corneal specialties and glaucoma management. Reichert Ocular Response Analyzer White Paper; 2005.

21. Congdon NG, Broman AT, Bandeen-Roche K, et al. Central corneal thickness and corneal hysteresis associated with glaucoma damage. Am J Ophthalmol 2006;141:868-75. 